\title{
Text Dependent Speaker Recognition using MFCC features and BPANN
}

\author{
Praveen N \\ Research Scholar \\ Department of Electronics \\ Audio and Image Research Laboratory \\ Cochin University of Science and Technology \\ Kochi, India 682022
}

\author{
Tessamma Thomas \\ Professor \\ Department of Electronics Cochin University of \\ Science and Technology \\ Kochi, India 682022
}

\begin{abstract}
Mel-Frequency Cepstral Coefficients are spectral feature which are widely used for speaker recognition and text dependent speaker recognition systems are the most accurate in voice based authentication systems. In this paper, a text dependent speaker recognition method is developed. MFCCs are computed for a selected sentence. The first 13 MFCCs are considered for each frames of duration $26 \mathrm{~ms}$ and each coefficient is clustered to a 5 element cluster centres and finally to a form a 65 element speech code vector for the entire speech. The speech code is trained using a multi-layer perceptron backpropagation gradient descent network and the network is tested for various test patterns. The performance is measured using FAR, FRR and EER parameters. The recognition rate achieved is $96.18 \%$ for a cluster size of 5 in each coefficient.
\end{abstract}

\section{General Terms}

Speaker Recognition, Artificial Neural Networks, Clustering

\section{Keywords}

Mel-Frequency Cepstral Coefficients, False Acceptance Rate, False Rejection Rate, Equal Error Rate

\section{INTRODUCTION}

Speaker verification/identification tasks are typically a pattern recognition problem. The important step in speaker recognition is the extraction of relevant features from the speech data that is used to characterize the speakers. There are two speaker dependent voice characteristics: high level and low level attributes [1][2]. Low level attributes related to the physical structure of the vocal tract are categorized as spectral characteristics, whereas high level attributes are prosody (pitch, duration, energy) or behavioral cues like dialect, word usage, conversation patterns, topics of conversation etc. There are two types of speaker recognition methods: text dependent and text independent. Text dependent speaker recognition method uses phoneme context information and hence high recognition accuracy is easily achieved. The text independent speaker recognition method does not require specially designed utterances and hence is user friendly. A general speaker recognition system consists of an enrolment phase and recognition phase.

In the speaker enrolment phase, speech samples are collected from the speakers to train their models. The collection of enrolled models is also called a speaker database. In the identification phase, a test sample from an unknown speaker is compared against the speaker database. Both phases include the feature extraction step to extract the speaker dependent characteristics from speech..

\section{METHODOLOGY}

The acoustic analysis based on MFCC has proved good results in speaker recognition. Also MFCC has proved to be good in confrontation with different variation such as noise, prosody, intonation. In this paper, speech samples of a given text are recorded for 40 speakers. 13 MFCCs are computed for about 40-45 frames of voiced speech samples. Vector Quantisation (VQ) based K-means clustering is done for the entire MFCC with respect to the cluster index. The code vector is trained using a discriminative classifier, multilayer perceptron with gradient descent backpropagation ANN. The system is tested with another data set of test patterns. The performance of the system is measured using false acceptance rate (FAR), false rejection rate (FRR) and equal error rate (EER). The result is compared with minimum distance based classifier. The methodology adopted is depicted in fig. 1. In the training phase, speech is recorded and pre-processed. MFC coefficients are computed and clustered using $K$-means clustering method. The speech code vector thus formed is trained to recognise using backpropagation neural network and stored. In the testing phase, the test speech samples are pre-processed and MFC coefficients are extracted and clustered. The test patterns are given to the trained neural network, which gives a decision of presence or absence of the speech pattern in the database.

\section{FEATURE EXTRACTION}

\subsection{Speech features}

According to Kinnunen [3], a vast number of features have been proposed for speaker recognition, such as:

- $\quad$ Spectral features

- Dynamic features

- $\quad$ Source features

- Supra segmental features

- High level features

Spectral features involve short time speech spectrum description and represent the physical characteristics of the vocal tract. Hence spectral feature relates to the physical behavior/characteristics of the vocal tract. 


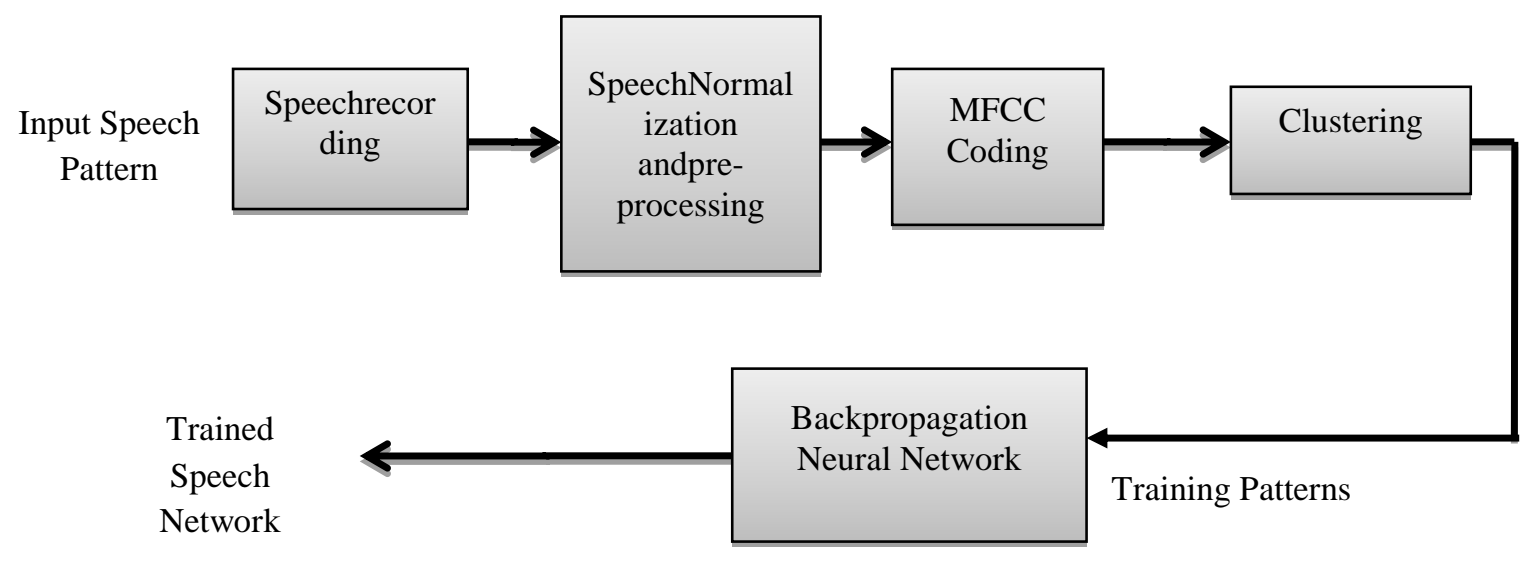

a) Training phase

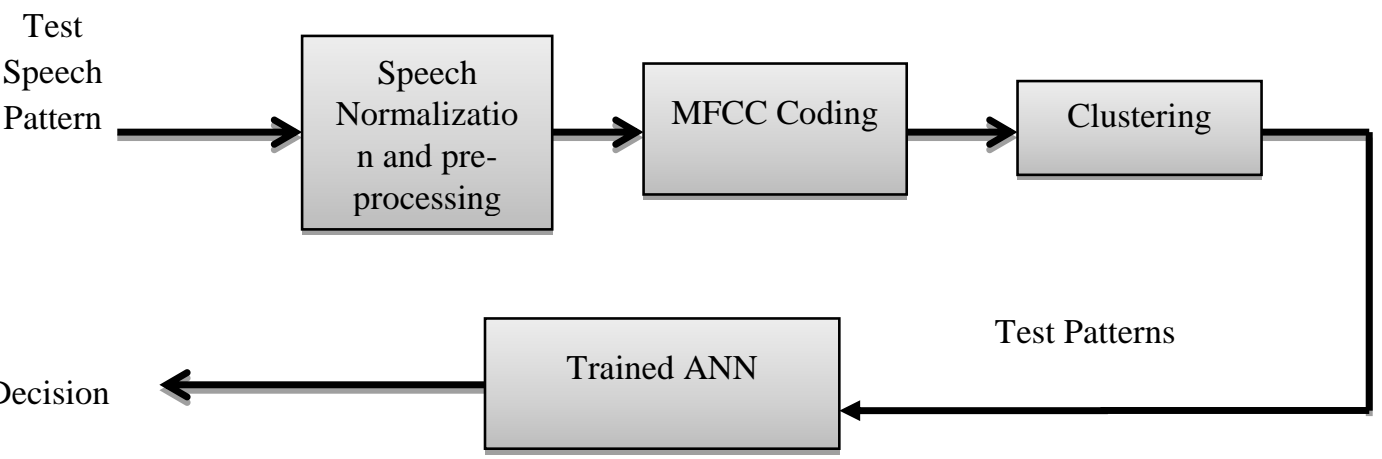

b) Testing phase

Fig. 1 Speaker Recognition steps

Table 1. Features for speaker recognition

\begin{tabular}{|c|c|}
\hline Feature Type & Examples \\
\hline Spectral features & $\begin{array}{l}\text { MFCC, LPCC, LSF, Long-term } \\
\text { average spectrum (LTAS), } \\
\text { Formant frequencies and } \\
\text { bandwidths }\end{array}$ \\
\hline Dynamic features & $\begin{array}{l}\text { Delta features, Modulation } \\
\text { frequencies, } \\
\text { autoregressive coefficients }\end{array}$ \\
\hline Source features & $F_{0}$ mean, Glottal Pulse shape \\
\hline Suprasegmental features & $\begin{array}{l}F_{0} \text { contours, Intensity contours, } \\
\text { Micro prosody }\end{array}$ \\
\hline High-level features & $\begin{array}{l}\text { Idiosyncratic word usage, } \\
\text { Pronunciation }\end{array}$ \\
\hline
\end{tabular}

Dynamic features relate to time evolution of spectral features. Source features are directly associated with glottal voice source. Supra segmental features span over several segments and high level feature refer to symbolic type of information. Table 1 gives the consolidation of the features for speaker recognition [3]. Since spectral features are the most common and accurate method available [4], a discussion on MFCC based spectral features is followed.

\subsection{Speech recording:}

In text dependent based speaker recognition, speech samples of the same text/sentence are to be recorded for all the speakers. The speakers are prompted to speak a sentence "open the door". Speech samples of 40 speakers are recorded in a laboratory environment using a high quality microphone with a DELL T7400 Intel Xeon based workstation computer under MATLAB 7.10.0.1 environment. The speech samples are subjected to the following operations as shown in fig. 1 . 


\subsection{Speech Normalization:}

In order to compensate for the changes in amplitude or energy of the speech recorded at different instances, the samples are normalized pre-processing [5]. Based on the maxima and minima of the signal, normalization has been carried out according to the equation:

$$
x_{i}=\lambda_{1}+\left(\lambda_{2}-\lambda_{1}\right)\left(\frac{z_{i}-z_{i}^{\min }}{z_{i}^{\max }-z_{i}^{\min }}\right)
$$

where $x_{i}$ is the normalized value of $z_{i}$, and $z_{i}^{\text {max }}$ and $z_{i}^{\text {min }}$ are the maximum and minimum values of $z_{i}$ in each sample. $\lambda_{1}$ and $\lambda_{2}$ can be set to 0.1 and 0.9 .

\subsection{Pre-processing:}

As a pre-processing step, pre-emphasis filtering is done for the speech signals. The speech is processed by a high-

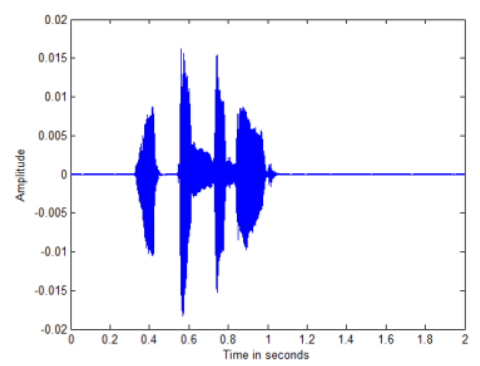

a)

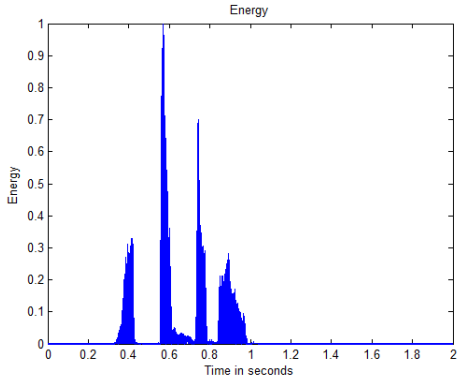

c) emphasis filter before input to the cepstrum analysis. This is required as the higher frequencies contain more speakerdependent information than the lower frequencies. A high pass filter with a transformation

$H(z)=1-a z^{-1}$

is used with $a=0.95$.

\subsection{Voiced region extraction:}

MFCCs of the voiced regions are computed in this work only to minimize the energy due to ambient noise. Hence voiced regions are isolated from the speech signal by considering the energy of the signal. The signal energy is computed and the voiced regions are extracted using the signal energy threshold. The voiced regions extracted for the normalized signal is shown in Fig. 2.

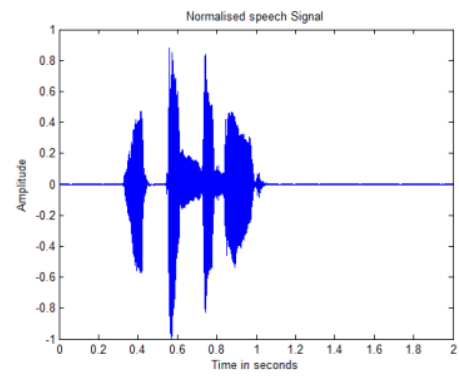

b)

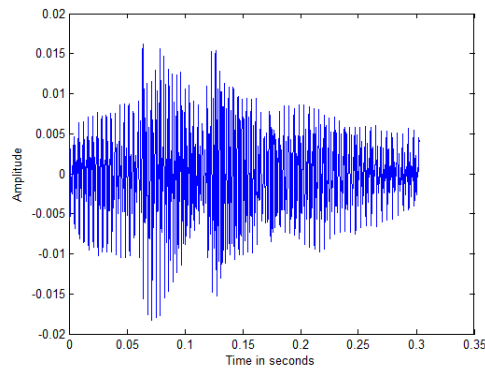

d)

Fig. 2 a) Speech signal b) Normalized speech signal c) Energy of the signal computed d) Voiced regions extracted

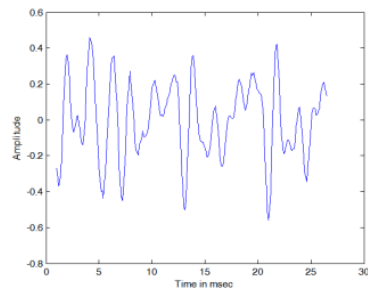

a)

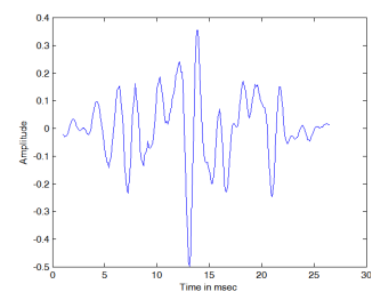

c)

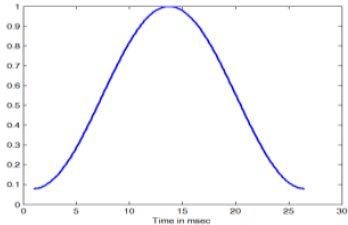

b)

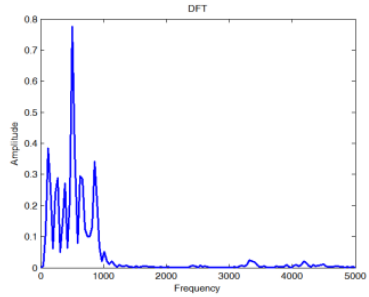

d)

Fig 3 a) A Speech Frame b) Hamming window c) Windowed Frame d) DFT of windowed frame 


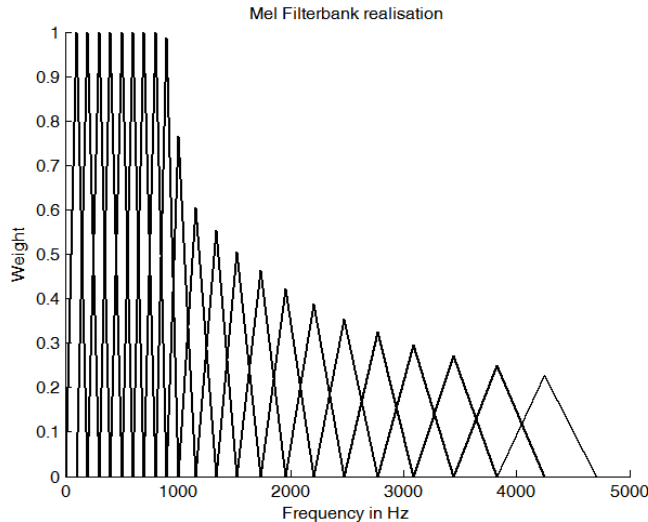

Fig. 4 Mel-filter Bank

\subsection{MFCC Computation:}

This method is a short-term spectral analysis method and speech signals are divided into short frames using the popular Hamming window of length 256 points and 256 point DFT is taken (Fig 3). The DFT of the frames is weighted by triangular Mel filterbanks (Fig. 4) and the mel-frequency spectrum at any analysis time $\hat{n}$ is computed as per the equation 4. The next step is to compute the DCT of the log of the magnitude of the filter outputs of each frame, which is the mel-frequency Cepstral coefficient of each frame, as given in equation 3.

$$
m f c c_{\hat{n}}[m]=\frac{1}{R} \sum_{r=1}^{R} \log \left(M F_{\hat{n}}[r]\right) \cos \left[\frac{2 \pi}{R}\left(r+\frac{1}{2}\right) m\right]
$$

where

$$
M F_{\hat{n}}=\frac{1}{A_{r}} \sum_{K=L_{r}}^{U_{r}}\left|V_{r}[K] X_{\hat{n}}[K]\right|^{2}
$$

and $\quad A_{r}=\sum_{L_{r}}^{U_{r}}\left|V_{r}[K]\right|^{2}$

Table 2 shows the MFCC of speech samples computed with $N_{m f c c}=13$ and $R=22$.

\subsection{Data clustering using K-means method:}

The MFCCs are calculated for about 40-45 frames with 13 coefficients for each frames. To reduce the size of the vector used for speaker recognition, clustering of each corresponding coefficients is done with respect to all frames limiting the size of speech vector of each speaker to a $5 \times 13=65$ element vector. $k$-means clustering, which is a squared mean clustering, is used for this. follows:

The k-Means Algorithm (LBG Algorithm) [6] is as

1. Design a 1-vector codebook which represents the centroid of the entire set of training vectors.
2. Double the size of the codebook by splitting each current codebook $\mathbf{y}_{\boldsymbol{n}}$ according to rule

$$
\begin{aligned}
& \mathbf{y}_{n}^{+}=\mathbf{y}_{n}(1+\varepsilon) \\
& \mathbf{y}_{n}^{-}=\mathbf{y}_{n}(1-\varepsilon)
\end{aligned}
$$

where $n$ varies from 1 to the current size of the codebook, and $\varepsilon$ is a splitting parameter (e.g. $\varepsilon=0.01$ )

3. Nearest-Neighbour Search: For each training vector, find the code word in the current codebook that is closest in terms of similarity measurement and assign that vector to the corresponding cell.

4. Centroid update: Update the code word in each cell using the centroid of the training vectors assigned to that cell.

5. Iteration 1: repeat steps 3 and 4 until the average distance falls below a pre-set threshold

6. Iteration 2: repeat steps 2,3 and 4 until a codebook size of $M$ is designed.

The LBG algorithm designs an M-vector codebook in stages. It starts first by designing 1-vector codebook, then uses a splitting technique on the code words to initialize the search for a 2-vector codebook, and continues the splitting process until the desired M-vector codebook is obtained. In this work, clustering of each of the 13 MFCC's is done for various cluster sizes ranging from 4 to 10 to find an optimum cluster size which will give the maximum performance during speaker recognition. Clustered speech codebook for cluster size 5 is given in Table 3 .

\section{NEURAL NETWORK DESIGN AND TRAINING}

\subsection{Network Architecture}

Multilayer perceptrons are feedforward neural networks trained using the backpropagation algorithm [7]. They can be used as binary classifiers for speaker verification to separate the speaker and non-speaker classes. In this work, a multilayer perceptron artificial neural network is trained with backpropagation algorithm. Design of a neural network involves the determination of number of neurons in the input layer, number of hidden layers and their size and determination of a proper activation function in the hidden and the output layer. In this work, the input layer size depends on the number of clustering coefficient. For example, a $5 \times 13$ codebook requires 65 numbers of input neurons. The number of hidden layer is set to 1 , and the size of number of hidden neurons is taken as 40 as per the criterion given by Masters [7] [8].The activation function taken for both the hidden and output layer is log-sigmoid. Fig. 5 shows the basic architecture of multilayer perceptron ANN for identifying 40 numbers of speakers with $65 \times 1$ input speech codebook, 1 hidden layer with 40 neurons and 40 output layer neurons. 
Table 2 MFCC of speech samples computed with $N_{m f c c}=13$ and $R=22$. (Only 28 frames are shown)

\begin{tabular}{|c|c|c|c|c|c|c|c|c|c|c|c|c|c|}
\hline & 1 & 2 & 3 & 4 & 5 & 6 & 7 & 8 & 9 & 10 & 11 & 12 & 13 \\
\hline $\begin{array}{c}\text { Frame } \\
1\end{array}$ & 2.30 & -4.03 & -6.24 & -0.86 & 0.21 & -1.28 & -2.17 & -1.05 & -0.27 & -1.28 & -1.00 & -0.37 & -0.13 \\
\hline 2 & 4.15 & -3.75 & -6.13 & -0.06 & 1.13 & -1.40 & -1.57 & 0.11 & -0.35 & -0.93 & -0.88 & 0.00 & 0.16 \\
\hline 3 & 3.39 & -4.40 & -6.95 & -0.90 & 0.35 & -2.10 & -1.57 & -0.67 & -0.67 & -1.11 & -1.10 & -0.19 & 0.02 \\
\hline 4 & 3.07 & -4.30 & -5.71 & -1.05 & -0.23 & -1.49 & -1.32 & -0.32 & -0.40 & -1.26 & -0.97 & -0.06 & 0.23 \\
\hline 5 & 3.86 & -4.06 & -6.84 & -1.47 & -0.42 & -2.69 & -1.61 & -0.15 & -1.11 & -1.39 & -1.00 & -0.55 & -0.13 \\
\hline 6 & 3.09 & -3.46 & -5.52 & -1.99 & -1.29 & -1.93 & -1.72 & -0.35 & -0.43 & -1.04 & -1.42 & -0.99 & -0.41 \\
\hline 7 & 1.57 & -0.36 & -3.65 & -1.15 & 0.47 & -0.73 & -1.18 & -0.03 & 0.06 & -0.41 & -1.47 & -1.20 & -0.38 \\
\hline 8 & -0.82 & 0.01 & -2.03 & 0.18 & 0.85 & -0.18 & -0.06 & 0.28 & -0.10 & -0.09 & -1.11 & -1.05 & -0.33 \\
\hline 9 & -3.68 & 0.25 & -0.77 & 0.08 & 1.44 & 0.07 & -0.20 & 0.15 & 0.13 & 0.10 & -0.16 & -0.17 & 0.23 \\
\hline 10 & -4.00 & 0.09 & -0.87 & -0.74 & 0.41 & -0.21 & -0.42 & 0.09 & 0.39 & -0.39 & -0.48 & -0.34 & -0.21 \\
\hline 11 & -4.84 & -0.04 & -1.64 & -1.47 & -0.28 & -0.02 & 0.61 & 0.55 & 0.44 & 0.06 & -0.87 & -0.66 & -0.48 \\
\hline 12 & -5.23 & -0.60 & -1.82 & -1.65 & -1.40 & -0.61 & 0.47 & 0.58 & 0.08 & -0.81 & -0.39 & -0.56 & -0.47 \\
\hline 13 & -6.51 & -1.09 & -1.91 & -1.38 & -1.07 & 0.02 & 0.14 & 0.25 & 0.03 & -0.92 & -0.55 & -0.51 & -0.40 \\
\hline 14 & \begin{tabular}{|l|}
-7.09 \\
\end{tabular} & -0.96 & -1.03 & -0.58 & -0.37 & -0.12 & -0.06 & -0.35 & 0.26 & 0.34 & 0.24 & -0.51 & -0.25 \\
\hline 15 & -1.80 & -2.74 & -1.77 & -0.52 & -2.18 & -0.28 & 0.42 & -0.62 & 0.12 & 0.69 & -0.30 & -0.85 & 0.15 \\
\hline 16 & 2.79 & -5.47 & -2.16 & -0.19 & -1.91 & -0.68 & 0.69 & 0.18 & -0.72 & -0.29 & 0.20 & -0.14 & 0.54 \\
\hline 17 & 1.59 & -6.71 & 0.10 & -0.84 & -0.94 & 0.43 & 0.55 & -0.14 & -1.06 & -0.24 & 0.19 & -0.49 & 0.57 \\
\hline 18 & -2.33 & -6.50 & 0.27 & -2.61 & -1.54 & -0.35 & 0.56 & -1.80 & -1.15 & -0.52 & 0.09 & -0.44 & 0.08 \\
\hline 19 & -2.79 & -6.27 & 0.51 & -3.24 & -0.93 & -0.79 & 0.83 & -2.56 & -0.25 & -0.33 & -0.61 & -0.13 & 0.10 \\
\hline 20 & -2.74 & -5.79 & -0.35 & -3.38 & -1.96 & -1.21 & 0.34 & -2.53 & -0.66 & 0.15 & -1.57 & -0.32 & 0.03 \\
\hline 21 & -1.22 & -3.94 & 0.59 & -2.65 & -2.87 & -0.87 & 0.22 & -1.08 & -0.94 & 0.23 & -1.13 & -0.27 & 0.19 \\
\hline 22 & 0.29 & -2.44 & -1.00 & -2.67 & -3.62 & -0.36 & -0.03 & -1.92 & -1.08 & -0.10 & -1.11 & -0.53 & 0.52 \\
\hline 23 & -0.34 & -1.36 & -0.87 & -3.09 & -4.26 & -0.49 & -0.04 & -2.21 & -1.51 & -0.82 & -1.39 & -0.48 & 0.58 \\
\hline 24 & 1.13 & 0.44 & -0.52 & -2.48 & -3.38 & -0.31 & 0.34 & -1.73 & -0.78 & -0.14 & -1.45 & -0.51 & 0.92 \\
\hline 25 & 0.61 & -0.12 & -0.99 & -3.02 & -3.64 & -0.31 & 0.14 & -1.82 & -1.05 & -0.40 & -1.47 & -0.66 & 0.58 \\
\hline 26 & -0.05 & 0.42 & -0.81 & -3.48 & -4.34 & -0.71 & -0.11 & -2.23 & -1.79 & -0.33 & -1.48 & -1.11 & 0.73 \\
\hline 27 & 0.74 & 1.63 & -0.57 & -2.38 & -3.36 & -0.99 & -0.38 & -1.16 & -0.81 & -0.26 & -1.74 & -0.53 & 0.92 \\
\hline 28 & 0.40 & 2.54 & -1.07 & -3.27 & -3.36 & -1.43 & -0.76 & -0.98 & -0.83 & -0.66 & -1.76 & -0.79 & 0.14 \\
\hline
\end{tabular}

Table 3. $k$-means clustered MFCC of speech samples computed with 5 cluster centers for each coefficient along each column MFCC's per frame $\rightarrow$ (13)

\begin{tabular}{|c|c|c|c|c|c|c|c|c|c|c|c|c|}
\hline 1 & 2 & 3 & 4 & 5 & 6 & 7 & 8 & 9 & 10 & 11 & 12 & 13 \\
\hline 3.39 & 1.53 & 0.20 & -1.70 & -4.87 & 1.72 & 0.09 & -1.22 & -3.52 & -5.63 & 0.27 & -0.82 & -2.07 \\
\hline-3.63 & -6.23 & 0.17 & -0.52 & -1.13 & -1.99 & -2.99 & 1.14 & 0.02 & -1.20 & -2.50 & -3.65 & 0.15 \\
\hline-0.30 & -0.74 & -1.23 & -1.86 & 0.64 & 0.26 & -0.19 & -1.09 & -1.73 & 0.41 & 0.00 & -0.50 & -1.21 \\
\hline-2.13 & 0.17 & -0.27 & -0.76 & -1.22 & -2.24 & 0.98 & 0.48 & 0.06 & -0.39 & -1.10 & 0.18 & -0.45 \\
\hline-1.04 & -1.43 & -2.03 & 0.70 & 0.46 & -0.22 & -0.58 & -1.07 & 0.85 & 0.56 & 0.10 & -0.17 & -0.40 \\
\hline
\end{tabular}




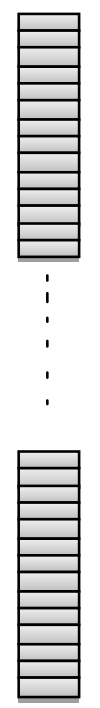

$65 \times 1$

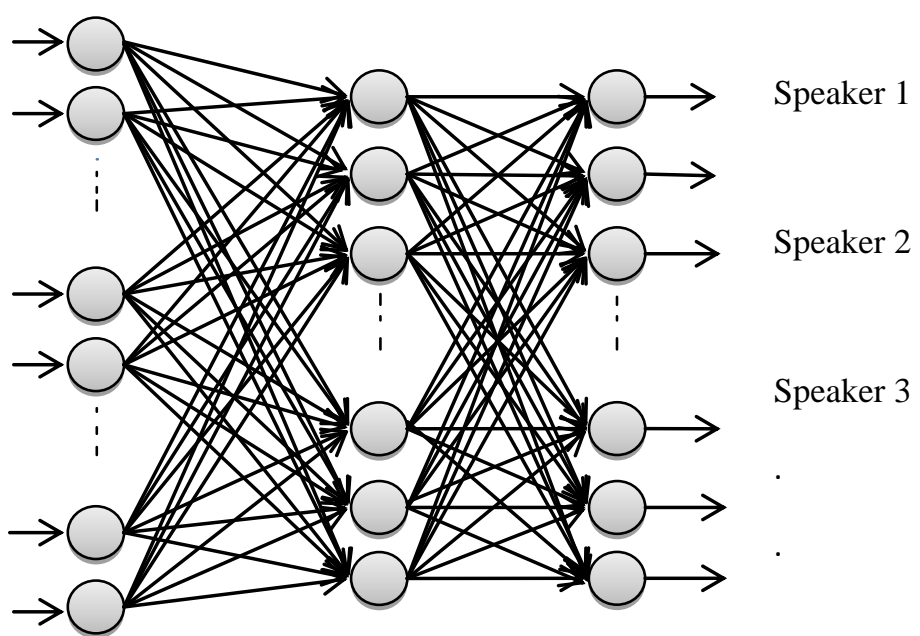

40 Hidden layer Neurons

40 Output Neurons

Fig.5 Neural Network Architecture for Speaker Recognition

\subsection{Data Normalisation:}

Before giving the data for training to the network, normalization is done as per equation 1 with $\boldsymbol{\lambda}_{\mathbf{1}}=$ 0.1 and $\lambda_{2}=0.9$ to avoid saturation of the sigmoid function leading to slow or no learning [9]. For each speaker 5 speech samples of the same sentence of duration 2 seconds have been recorded in the laboratory environment and stored.
The clustered MFCC computed is arranged as a column vector for each speech sample. Speech feature vectors of all the 40 speakers are normalized and arranged as a matrix of size $65 \times 200$ (for a cluster size of 5), with column 1-5 corresponding to speaker 1, column 6-10 correspond to speaker 2 and likewise (Table. 4).

Table 4. Database for training. (Column 1-5 belongs to speaker 1, column 6-10 belongs to speaker 2. Only 14 elements of speech vector are shown)

\begin{tabular}{|c|c|c|c|c|c|c|c|c|c|}
\hline 1 & 2 & 3 & 4 & 5 & 6 & 7 & 8 & 9 & 10 \\
\hline 0.357 & 0.263 & 0.389 & 0.287 & 0.369 & 0.432 & 0.467 & 0.493 & 0.479 & 0.465 \\
\hline 0.142 & 0.101 & 0.156 & 0.096 & 0.146 & 0.256 & 0.285 & 0.362 & 0.281 & 0.323 \\
\hline-0.072 & -0.093 & -0.099 & -0.029 & -0.027 & 0.137 & 0.112 & 0.180 & 0.082 & 0.055 \\
\hline-0.292 & -0.236 & -0.415 & -0.260 & -0.303 & -0.130 & -0.202 & -0.134 & -0.145 & -0.174 \\
\hline-0.471 & -0.405 & -0.577 & -0.527 & -0.562 & -0.372 & -0.448 & -0.368 & -0.446 & -0.437 \\
\hline 0.139 & 0.149 & 0.239 & 0.146 & 0.101 & 0.230 & 0.131 & 0.292 & 0.246 & 0.269 \\
\hline 0.039 & 0.055 & 0.202 & 0.083 & 0.026 & 0.139 & 0.010 & 0.179 & 0.133 & 0.173 \\
\hline-0.052 & -0.044 & 0.044 & -0.017 & -0.061 & 0.028 & -0.150 & -0.037 & -0.058 & -0.040 \\
\hline-0.143 & -0.140 & -0.104 & -0.141 & -0.178 & -0.133 & -0.303 & -0.202 & -0.228 & -0.208 \\
\hline-0.259 & -0.294 & -0.259 & -0.240 & -0.288 & -0.335 & -0.500 & -0.410 & -0.405 & -0.387 \\
\hline 0.158 & 0.244 & 0.311 & 0.273 & 0.196 & 0.319 & 0.176 & 0.203 & 0.250 & 0.151 \\
\hline 0.110 & 0.149 & 0.178 & 0.207 & 0.138 & 0.133 & 0.101 & 0.148 & 0.121 & 0.059 \\
\hline 0.052 & 0.080 & 0.059 & 0.099 & 0.070 & 0.058 & 0.020 & 0.085 & 0.063 & -0.011 \\
\hline-0.012 & 0.018 & -0.018 & 0.009 & -0.025 & -0.014 & -0.048 & -0.019 & -0.002 & -0.110 \\
& & & & & & & & & \\
\hline
\end{tabular}




\subsection{ANN training:}

The BPANN is trained as per the backpropagation algorithm [9] with the input neuron size as a function of cluster size. The network used is gradient decent backpropagation with adaptive learning rate for 1000 epochs. The performance and gradient is set as $1 \mathrm{e}-08$ and $1 \mathrm{e}-10$.

For each input cluster sizes, neural networks are trained and the corresponding net is stored and prepared for testing with a test database. The test database consists of known and unknown speakers which are classified as genuine and impostor speakers. 200 speech samples in the test database are tested and the false acceptance rate (FAR) and false rejection rate (FRR) of the system are estimated.

\section{IMPLEMENTATION}

\subsection{Database used}

In this work, speech samples of persons are recorded using a high quality microphone under MATLAB 7.10.0.1 environment. For each speaker a particular text "Open the door" is prompted to speak for about 2 seconds. The sampling frequency used to record is $10 \mathrm{~K} \mathrm{~Hz}$. For each speaker, 10 samples are stored with 5 samples are used for training phase and other 5 samples are taken for testing/validating the algorithm.

\subsection{Implementation}

Before extracting the features from the speech signal, each speech sample is normalized using equation 1 to set the signal amplitude within a desired range i.e., between 0.1 and 0.9 . Each speech sample is then subjected to a pre-emphasis filtering as per equation 2 with $a=0.95$. In the next step, the voiced regions in the filtered waveform are extracted using the energy computed from the signal amplitude. For energy $\geq$ 0.01 , the signal regions are sampled as voiced regions. The voiced regions extracted for a speech sample are shown in Fig. 2d.

MFCCs of speech signals are extracted using the equation 4 for the voiced regions. For this, speech signals are framed for about 256 samples (about $26 \mathrm{msec}$ ) with a frame overlap of
100 samples. FFT is computed for each samples and the spectrum is weighted 22 Triangular filter banks. MFCC of the frame is calculated for each frame and stored.

In this paper, MFCCs of speech samples are computed and clustered with clusters of different sizes, i.e., clusters of size $10,8,7,5$ and 4 using $k$-means clustering algorithm. Backpropagation based ANNs are designed for various input clusters. In this case a gradient descent based backpropagation with adaptive learning rate is selected for training. The number of hidden layer is set as 1 and the hidden and output neuron size is set as 40 , irrespective of the input neuron dimension. The network is trained with 40 speakers' speech samples for given set performance. The trained network is stored for testing/validation.

\subsection{Results and Discussion}

Out of 10 speech samples recorded for each speaker, 5 samples are taken for feature extraction and training. i.e., the input data size for training the network is of size $200 \times 65$ for a cluster size of 5. ANN is designed and trained for various input cluster sizes of 10, 8, 5 and 4. The False Acceptance Rate (FAR) and False Rejection Rate (FRR) of the system for various clusters are shown in Fig.8 and Fig.9. The Equal Error Rates (EERs) corresponding to each cluster size is shown in Table 5. It is seen that minimum EER is obtained for the cluster of size 5 . Hence cluster of size 5 can be considered as the best option for the recognition as the EER is approximately 0.0382 within a matching threshold of 0.152 0.167 . Thus the maximum genuine acceptance rate or recognition rate achieved for this system is $96.18 \%$. Also the mean-squared difference (using minimum distance classifier) between the testing and training vectors for various cluster sizes are calculated and the EER obtained with cluster size of 5 is about 0.1662 which is the minimum EER obtained among all the clusters. Thus only $83.38 \%$ of recognition rate can be obtained using minimum distance classifier based direct method. The comparison of neural network based and minimum distance based classifiers are shown in Table 6 from which it can be conclude that the neural network method can be adopted for speaker identification with minimum error as the EER is only $3.82 \%$ for a cluster size 5 .

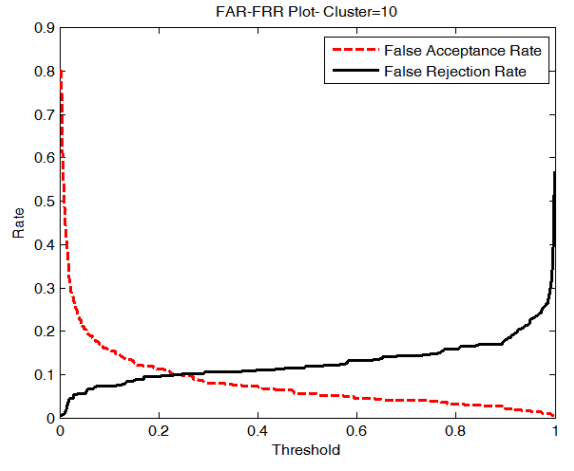

a)

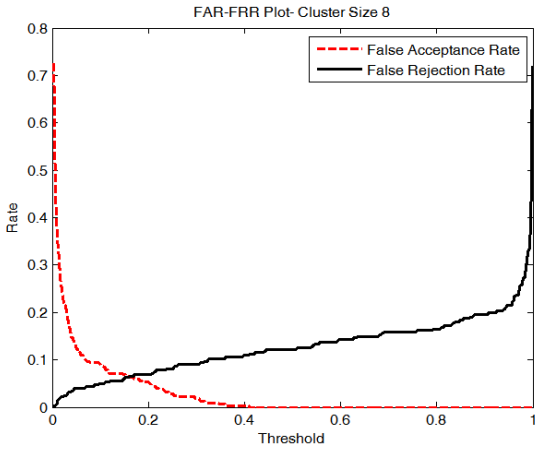

b)

Fig. 6 a) FAR-FRR plot for cluster size=10. EER=0.0997 at a Threshold =0.232 to 0.247

b) FAR-FRR plot for cluster size=8. EER=0.0639 at a Threshold of 0.1625 

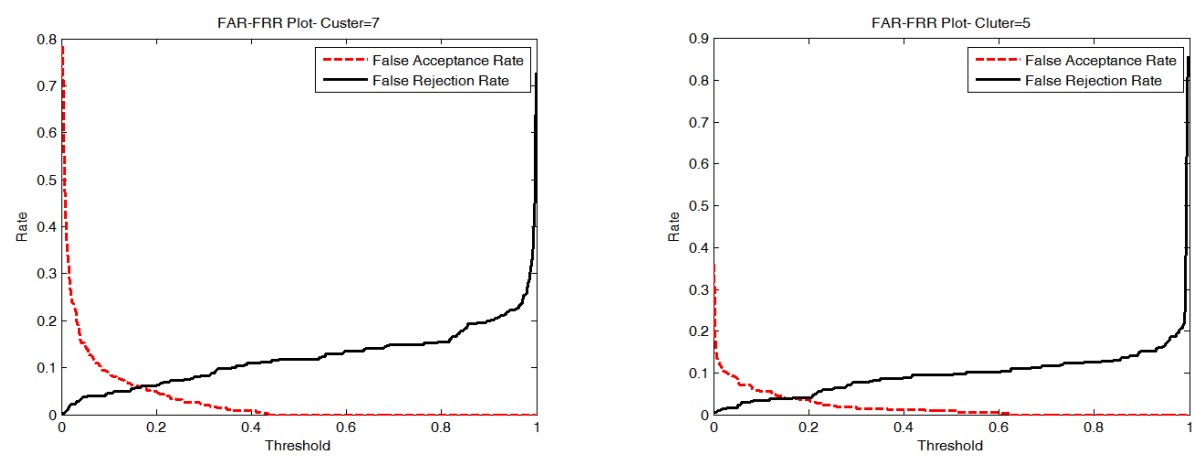

a)

b)

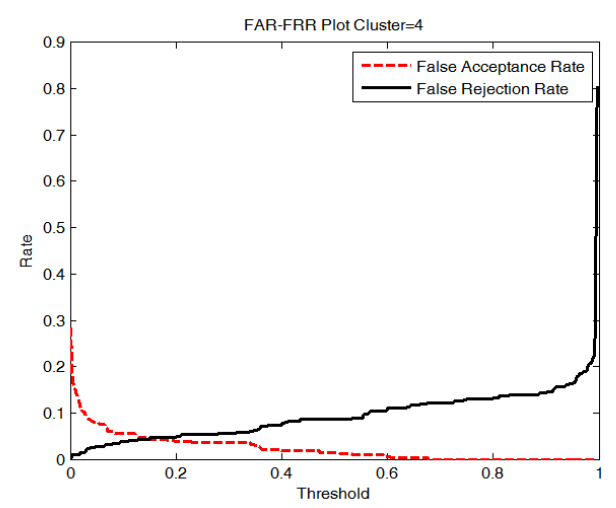

c)

Fig. 7a) FAR-FRR plot for cluster size=7. EER=0.0576 at aThreshold of 0.163-0.166

b) FAR-FRR plot for cluster size=5. EER=0.0382 at a Threshold of 0.152-0.167

c) FAR-FRR plot for cluster size $=4$. $E E R=0.0452$ at a Threshold of 0.1505

Table 5. Cluster Size and EER for ANN method

\begin{tabular}{|c|c|c|}
\hline Cluster Size & EER & Threshold Range \\
\hline 10 & 0.0997 & 0.2320 \\
\hline 8 & 0.0639 & 0.1625 \\
\hline 7 & 0.0576 & $0.163-0.166$ \\
\hline $\mathbf{5}$ & $\mathbf{0 . 0 3 8 2}$ & $\mathbf{0 . 1 5 2 - 0 . 1 6 7}$ \\
\hline 4 & 0.0452 & 0.1505 \\
\hline
\end{tabular}

Table 6. Comparison of EER for ANN and Euclidean

\begin{tabular}{|c|c|c|}
\hline \multirow{2}{*}{$\begin{array}{c}\text { Cluster } \\
\text { Size }\end{array}$} & \multicolumn{2}{|c|}{ distance classifier } \\
\cline { 2 - 3 } & $\begin{array}{c}\text { Euclidean } \\
\text { distance } \\
\text { method }\end{array}$ & $\begin{array}{c}\text { Neural Network } \\
\text { method }\end{array}$ \\
\hline 10 & 0.2456 & 0.0997 \\
\hline 8 & 0.2093 & 0.0639 \\
\hline 7 & 0.1812 & 0.0576 \\
\hline $\mathbf{5}$ & $\mathbf{0 . 1 6 6 2}$ & $\mathbf{0 . 0 3 8 2}$ \\
\hline 4 & 0.1753 & 0.0452 \\
\hline
\end{tabular}




\section{CONCLUSIONS}

In this paper speaker recognition technique based on spectral characteristics, mel-frequency cepstral coefficient, was developed. K-means clustering was done to minimize the codebook size. The recognition based on multilayer perceptron classifier and minimum distance Euclidean distance classifier was studied. The Euclidean distance classifier yield only $83.38 \%$ of recognition rate while the multilayer perceptron classifier yields $96.18 \%$ of recognition rate.

\section{REFERENCES}

[1] Thomas F. Quatieri, Discrete Time Signal Processing Principles and Practice, Pearson Education Inc.India.

[2] J. P. Campbell, Speaker recognition: A tutorial, Proc. IEEE, vol. 85, pp. 1437-1462, 1997.

[3] Tomi H. Kinnunen, Optimizing Spectral Feature Based Text-Independent Speaker Recognition, Academic Dissertation, University of Joensuu, 2005.
[4] P. Melmerstein and S.B. Davis, Comparison of parametric representations for monosyllabic word recognition in continuously spoken sentences, IEEE Trans Acoustic, Speech, and Signal Processing, vol 28, no. 4, pp. 357-366,1980.

[5] Hassoun, M. H, Fundamentals of Artificial Neural Networks. MIT Press, Cambridge, MA.

[6] Y.Linde, A.Buzo, and R.M.Gray, An algorithm for vector quantizer design, IEEE Trans. on Communications, vol. COM_28 (1), pp.84-96, Jan. 1980.

[7] I.A. Basheer, M. Hajmeer, Artificial neural networks: fundamentals, computing, design and application, Journal of microbiological methods 43 (2000) 3-31.

[8] Masters, T., 1994. Practical Neural Network Recipes in C11. Academic Press, Boston, MA

[9] Haykin S, Neural Networks: A Comprehensive Foundation, Macmillan, New York, 2004 